\title{
Histopathological Findings in Laparoscopic Sleeve Gastrectomy Specimens from Patients with Obesity in Saudi Arabia
}

\author{
Khaldoon Aljerian (iD \\ Forensic Medicine Unit, Department of Pathology, College of Medicine, King Saud University, Riyadh 12372, Saudi Arabia \\ Correspondence should be addressed to Khaldoon Aljerian; khaldoonaljerian@gmail.com
}

Received 3 November 2017; Revised 9 February 2018; Accepted 28 February 2018; Published 3 April 2018

Academic Editor: Per Hellström

Copyright (c) 2018 Khaldoon Aljerian. This is an open access article distributed under the Creative Commons Attribution License, which permits unrestricted use, distribution, and reproduction in any medium, provided the original work is properly cited.

\begin{abstract}
Laparoscopic sleeve gastrectomy is a bariatric surgical procedure performed in patients with morbid obesity that provides the opportunity to review histopathological changes. The aim of this study was to characterize resected gastric specimens obtained from a Saudi patient population at a single center for a prospectively maintained database of laparoscopic sleeve gastrectomy cases. The histopathological findings from all patients undergoing laparoscopic sleeve gastrectomies at King Khalid University Hospital between 2010 and 2015 were retrospectively reviewed. Of the 602 cases reviewed, the majority $(83.4 \%$ [ $n=502])$ exhibited chronic gastritis, whereas $22.3 \%(n=134)$ involved Helicobacter pylori infections with active gastritis, $1 \%(n=6)$ had intestinal metaplasia, and one case $(0.17 \%)$ revealed gastric adenocarcinoma. As the findings revealed conditions that are treatable, I highly recommend histological examinations of all sleeve gastrectomy specimens from a Saudi patient population.
\end{abstract}

\section{Introduction}

Obesity and its various comorbidities currently comprise the most serious, most prevalent, and most challenging health concerns worldwide $[1,2]$. For patients with severe obesity, high body mass indexes (BMIs), serious comorbidities, and extremely poor qualities of life, bariatric (weight loss) surgery can provide a substantial health benefit despite the operative risks [3]. Among the currently available bariatric surgical procedures and techniques, the most favored is laparoscopic sleeve gastrectomy (LSG) as either the sole treatment for obesity or as a component of complex bariatric surgical interventions [4]. The restrictive and metabolic effects of LSG result from the vertical resection of a large portion of the stomach, including the fundus [5-8]. These resection specimens are the sources for vast amounts of published data on histopathological changes in patients from developed countries and may provide a basis for monitoring long- and short-term effects of the operation. Thus, it is highly advised that bariatric/metabolic surgeons collect these specimens to provide further data on LSG.

Obesity and its comorbid conditions are widely prevalent in Saudi Arabia [9]. The World Health Organization reported that $23 \%$ and $36 \%$ of male and female Saudi individuals, respectively, over the age of 15 years were classified with obesity in 2010 [10]. The increasing incidence of obesity in this population has placed Saudi Arabia among the list of nations where bariatric surgery is becoming popular [11]. In this study, the histopathological findings in resected gastric specimens from patients undergoing LSG at a single center in Riyadh, Saudi Arabia, were examined for comparison with other published findings and to provide a basis for future guidelines for preoperative assessment and the design of more holistic treatment decisions.

\section{Materials and Methods}

This was a retrospective study conducted at the Department of Pathology, King Khalid University Hospital (KKUH), Saudi Arabia, after receiving approval from the institutional ethics committee. All patients undergoing LSGs for the treatment of morbid obesity between 2010 and 2015 were included in the study. Cases with partial gastrectomies for all other reasons were excluded.

Patient characteristics such as age, sex, and initial BMI were retrieved from clinical records. The findings of gross 


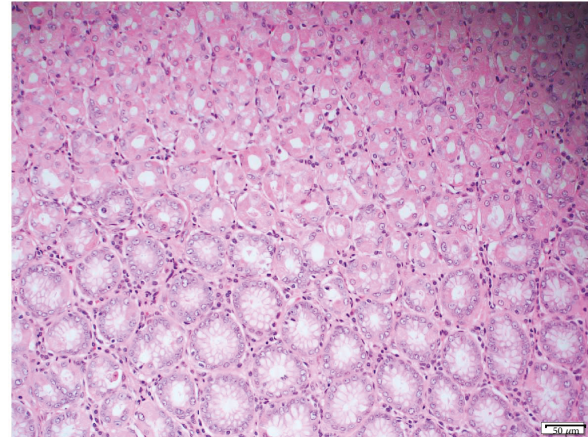

(a)

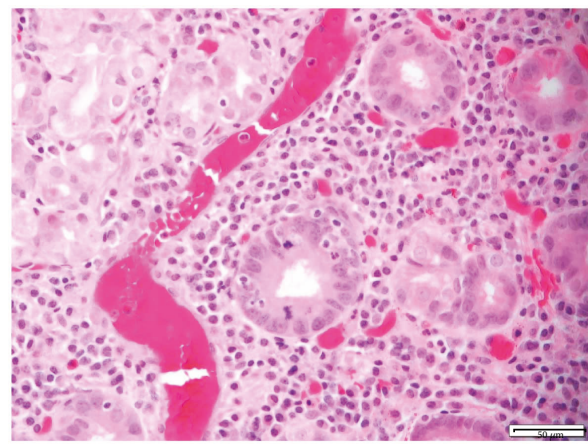

(c)

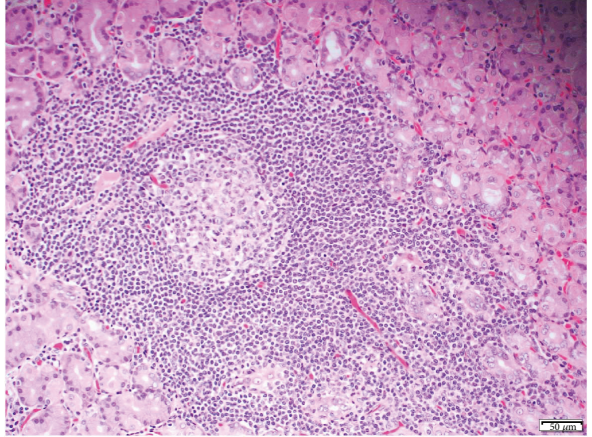

(b)

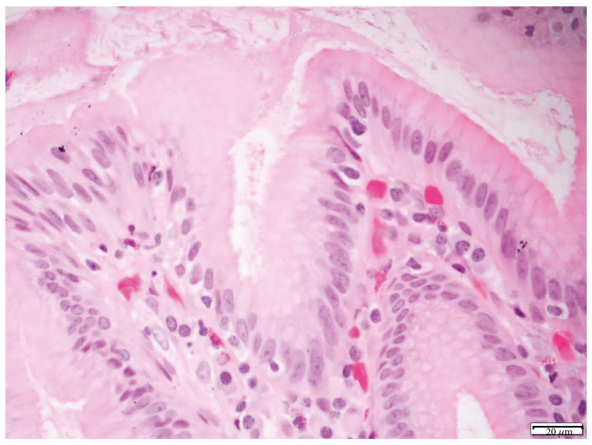

(d)

FIGURE 1: (a) Normal stomach mucosal epithelium and glands, paraffin embedded, H\&E stained, $\times 200$. (b) Chronic inflammation of stomach mucosal epithelium with mucosal follicle, paraffin embedded, H\&E stained, $\times 200$. (c) Acute and chronic inflammation of stomach mucosal epithelium with neutrophil infiltration of gland, paraffin embedded, H\&E stained, $\times 400$. (d) Stomach mucosal epithelium with $H$. pylori-like organisms on the surface, paraffin embedded, H\&E stained, $\times 600$.

and histopathological examinations in the final biopsy reports were retrospectively reviewed in addition to the mentioned diagnosis. For a comprehensive analysis, the cases were categorized into the following groups on the basis of the biopsy report findings: (i) normal histology of stomach, (ii) chronic active gastritis with Helicobacter pylori infection, (iii) chronic gastritis alone, (iv) follicular lymphoid hyperplasia, (v) intestinal metaplasia, (vi) dysplasia/carcinoma in situ, (vii) carcinoma, and (viii) other pathological entities (such as polyps and leiomyoma). The data were entered and analyzed using the Statistical Package for Social Sciences (SPSS) version 20 for Windows. Simple descriptive statistics were applied for discrete variables such as sex and diagnosis. The means and standard errors and 95\% confidence intervals (CIs) were calculated for continuous variables, such as age and BMI.

\section{Results}

Data from 602 patients were available for the retrospective analysis. The mean age of the patients was $31.36 \pm 0.78$ years (95\% CI, 29.82-32.90 years), including 266 (44.2\%) male and 336 (55.8\%) female patients (male/female ratio, 4:5). Gross examinations of surgical specimens revealed that most (580/602 [96.4\%]) had smooth, unremarkable outer surfaces, whereas congestion, hemorrhage, nodularity, flattening, sloughing, and edema were found in 60 (10\%), 42 (7\%), 4
TABle 1: Histological diagnoses identified by examining 602 patients.

\begin{tabular}{lcc}
\hline Histological diagnosis & Percentage & Frequency \\
\hline Chronic gastritis & $83.4 \%$ & 502 \\
Active gastritis & $22.3 \%$ & 134 \\
H. pylori & $22.3 \%$ & 134 \\
Intestinal metaplasia & $1 \%$ & 6 \\
Adenocarcinoma & $0.17 \%$ & 1 \\
\hline
\end{tabular}

(0.7\%), $4(0.7 \%), 2(0.3 \%)$, and $2(0.3 \%)$ specimens, respectively. The mean wall thickness was $5.76 \pm 0.21 \mathrm{~mm}(95 \%$ CI, 5.34-6.18 mm).

Histological examinations indicated that the majority (83.4\% [502/602]) of specimens had submucosal lymphocytic and plasmacytic infiltrates and thus constituted the diagnoses of chronic gastritis (Figure 1(b)). The second most frequent findings were the presence of $H$. pylori in the mucosal surfaces and crypts (22.3\% [134/502]) and signs of active inflammation evidenced by the presence of neutrophils in the mucosal lining or the vicinity $(22.3 \%$ [134/502]) for the diagnoses of active gastritis (Figures 1(c) and 1(d)). Most strikingly, 6 (1\%) specimens were from patients with premalignant conditions, that is, intestinal metaplasia, and gastric adenocarcinoma was identified in $1(0.17 \%)$ specimen (Table 1). 


\section{Discussion}

As LSG is commonly used to treat obesity at KKUH, there is an opportunity to learn from the histopathological findings in the resected stomach specimens of these patients. There was a high prevalence of chronic gastritis $(83.4 \%)$ and a moderate prevalence of active gastritis (22.3\%), including cases with $H$. pylori infection. These findings are in line with those of Almazeedi et al. [12], who examined 656 LSG specimens from similarly aged Arab patients and showed that $74.4 \%$ had features of chronic gastritis, with $9.6 \%$ showing follicular gastritis and $1.8 \%$ with atrophic gastritis, but with only $7.3 \%$ of cases with $H$. pylori infections. They also reported four cases $(0.6 \%)$ with gastric polyps, three cases $(0.5 \%)$ with granulomatous disease, and one case $(0.2 \%)$ each with a gastrointestinal stromal tumor, a gastrointestinal autonomic nerve tumor, intestinal metaplasia, collagenous gastritis, and crypt cell apoptosis [12]. In the present study, $1 \%$ of specimens had premalignant intestinal metaplasia, with one case of gastric adenocarcinoma. Similarly, a study of 27 patients with obesity undergoing LSG at another hospital in Saudi Arabia (King Fahd University Hospital, Al-Khobar) showed that the majority (74\%) had chronic gastritis, with $11.1 \%$ showing signs of active gastritis associated with H. pylori infection [13]. However, the higher prevalence of $H$. pylori infection, in the present study, is in line with previous studies in Saudi Arabia [14-16].

A study of 33 Turkish patients with morbid obesity undergoing LSG by Gündoğan et al. [17] revealed interstitial lymphocytic infiltration (63.6\%), hyperplasia of lymphoid follicles in the lamina propria $(60.7 \%)$, and microvesiculation/dilatation of parietal cells (57.6\%). A retrospective review of 48 LSG specimens from a U.S. hospital by Raess et al. [18] identified cases (8.4\%) that warranted clinical follow-ups, including those with $H$. pylori-associated gastritis (observed in 5.2\% of all cases and in $33.3 \%$ of cases of gastritis), autoimmune gastritis with microcarcinoid formation, necrotizing vasculitis, and intestinal metaplasia; neoplasms were identified at laparoscopy in 2 additional cases $(0.8 \%)$. Another study of 125 U.S. patients undergoing LSG for obesity by Clapp [19] found that $49.7 \%$ of the resected specimens had histopathological findings, including acute $(n=4)$ and chronic $(n=61)$ gastritis, follicular lymphoid hyperplasia $(n=11)$, leiomyoma $(n=1)$, and fundic polyps $(n=2)$. Behrens et al. [20] followed up 34 Canadian patients (mean age, 48 years) with obesity undergoing LSG and found $H$. pylori-associated gastritis in only $2(6 \%)$ patients and small gastrointestinal stromal tumors in $1(3 \%)$ patient. A higher prevalence of $H$. pylori (33.3\%) in association with chronic gastritis with inflammatory activity was reported in a Brazilian study by Onzi et al. [21].

In accordance with the high prevalence of gastritis among populations with obesity, the majority of histopathology results after LSG have an element of chronic gastritis. However, previous studies also show that at least a few cases undergoing LSG are found to harbor clinically significant pathologies that necessitate alterations in postoperative management [12]. A recent study by AbdullGaffar et al. [22] suggests that histological examination of LSG specimens from selected cases is more cost-effective than microscopic examination of all of them. However, the high prevalence of $H$. pylori infection and identification of premalignant and rare malignant cases by the present and other studies indicates that histological examinations of all specimens are warranted. This is particularly relevant for the Saudi population, for which life-threatening findings were noted that required an alteration of patient management.

\section{Conclusions}

I recommend routine histological examination of gastric specimens from patients after LSG for obesity across Saudi Arabia, considering the high prevalence of $H$. pylori infection in the population. We invite colleagues to add their experiences at various centers so that national guidelines may be formulated to address the preoperative and operative management of such patients and tackle these so-called "unanticipated findings" effectively.

\section{Conflicts of Interest}

The authors declare that they have no conflicts of interest.

\section{Acknowledgments}

The author thanks the College of Medicine, King Saud University (College of Medicine Research Center [CMRC]) for their support.

\section{References}

[1] A. M. Prentice, "The emerging epidemic of obesity in developing countries," International Journal of Epidemiology, vol. 35, no. 1, pp. 93-99, 2006.

[2] B. A. Swinburn, G. Sacks, K. D. Hall et al., "The global obesity pandemic: shaped by global drivers and local environments," Lancet, vol. 378, no. 9793, pp. 804-814, 2011.

[3] R. J. Rosenthal and I. S. Panel, "International sleeve gastrectomy expert panel consensus statement: best practice guidelines based on experience of $>12,000$ cases," Surgery for Obesity and Related Diseases, vol. 8, no. 1, pp. 8-19, 2012.

[4] I. Kehagias, S. N. Karamanakos, M. Argentou, and F. Kalfarentzos, "Randomized clinical trial of laparoscopic Roux-en-Y gastric bypass versus laparoscopic sleeve gastrectomy for the management of patients with BMI $<50 \mathrm{~kg} / \mathrm{m}^{2}$," Obesity Surgery, vol. 21, no. 11, pp. 1650-1656, 2011.

[5] M. Gagner, M. Deitel, A. L. Erickson, and R. D. Crosby, "Survey on laparoscopic sleeve gastrectomy (LSG) at the Fourth International Consensus Summit on Sleeve Gastrectomy," Obesity Surgery, vol. 23, no. 12, pp. 2013-2017, 2013.

[6] N. Bülbüller, A. Aslaner, O. Z. Öner et al., "Comparison of four different methods in staple line reinforcement during laparoscopic sleeve gastrectomy," International Journal of Clinical and Experimental Medicine, vol. 6, no. 10, pp. 985-990, 2013.

[7] E. E. Frezza, "Laparoscopic vertical sleeve gastrectomy for morbid obesity. The future procedure of choice?," Surgery Today, vol. 37, no. 4, pp. 275-281, 2007.

[8] M. Rubin, R. T. Yehoshua, M. Stein et al., "Laparoscopic sleeve gastrectomy with minimal morbidity early results in 120 
morbidly obese patients," Obesity Surgery, vol. 18, no. 12, pp. 1567-1570, 2008.

[9] M. El Mouzan, P. Foster, A. Al Herbish et al., "Prevalence of overweight and obesity in Saudi children and adolescents," Annals of Saudi Medicine, vol. 30, no. 3, pp. 203-208, 2010.

[10] M. Badran and I. Laher, "Obesity in Arabic-speaking countries," Journal of Obesity, vol. 2011, Article ID 686430, 9 pages, 2011.

[11] H. Buchwald and D. M. Oien, "Metabolic/bariatric surgery worldwide 2011," Obesity Surgery, vol. 23, no. 4, pp. 427436, 2013.

[12] S. Almazeedi, S. Al-Sabah, A. Al-Mulla et al., "Gastric histopathologies in patients undergoing laparoscopic sleeve gastrectomies," Obesity Surgery, vol. 23, no. 3, pp. 314-319, 2013.

[13] A. Ahmed, "Histopathological spectrum of sleeve gastrectomy in King Fahd University Hospital, Alkhobar, Saudia Arabia," Khyber Medical University Journal, vol. 4, no. 2, pp. 39-44, 2012.

[14] D. H. Akbar and A. T. Eltahawy, "Helicobacter pylori infection at a university hospital in Saudi Arabia: prevalence, comparison of diagnostic modalities and endoscopic findings," Indian Journal of Pathology and Microbiology, vol. 48, no. 2, pp. 181185,2005

[15] A. Albawardi, S. Almarzooqi, and F. C. Torab, "Helicobacter pylori in sleeve gastrectomies: prevalence and rate of complications," International Journal of Clinical and Experimental Medicine, vol. 6, no. 2, pp. 140-143, 2013.

[16] M. A. Khan and H. O. Ghazi, "Helicobacter pylori infection in asymptomatic subjects in Makkah, Saudi Arabia," Journal of the Pakistan Medical Association, vol. 57, no. 3, pp. 114-117, 2007.

[17] M. Gündoğan, N. Çalli Demirkan, K. Tekin, and H. Aybek, "Gastric histopathological findings and ghrelin expression in morbid obesity morbid/Obezitede gastrik histopatolojik bulgular ve ghrelin ekspresyonu," Turk Patoloji Dergisi, vol. 29, no. 1, pp. 19-26, 2013.

[18] P. W. Raess, M. Baird-Howell, R. Aggarwal, N. N. Williams, and E. E. Furth, "Vertical sleeve gastrectomy specimens have a high prevalence of unexpected histopathologic findings requiring additional clinical management," Surgery for Obesity and Related Diseases, vol. 11, no. 5, pp. 1020-1023, 2015.

[19] B. Clapp, "Histopathologic findings in the resected specimen of a sleeve gastrectomy," Journal of the Society of Laparoendscopic Surgeons, vol. 19, no. 1, article e2013.00259, 2015.

[20] C. Behrens, B. Q. Tang, and B. J. Amson, "Early results of a Canadian laparoscopic sleeve gastrectomy experience," Canadian Journal of Surgery, vol. 54, no. 2, pp. 138-143, 2011.

[21] T. R. Onzi, A. J. d’Acampora, F. M. de Araújo et al., "Gastric histopathology in laparoscopic sleeve gastrectomy: pre-and post-operative comparison," Obesity Surgery, vol. 24, no. 3, pp. 371-376, 2014.

[22] B. AbdullGaffar, L. Raman, A. Khamas, and F. AlBadri, "Should we abandon routine microscopic examination in bariatric sleeve gastrectomy specimens?," Obesity Surgery, vol. 26, no. 1, pp. 105-110, 2016. 


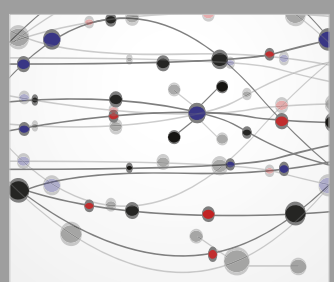

The Scientific World Journal
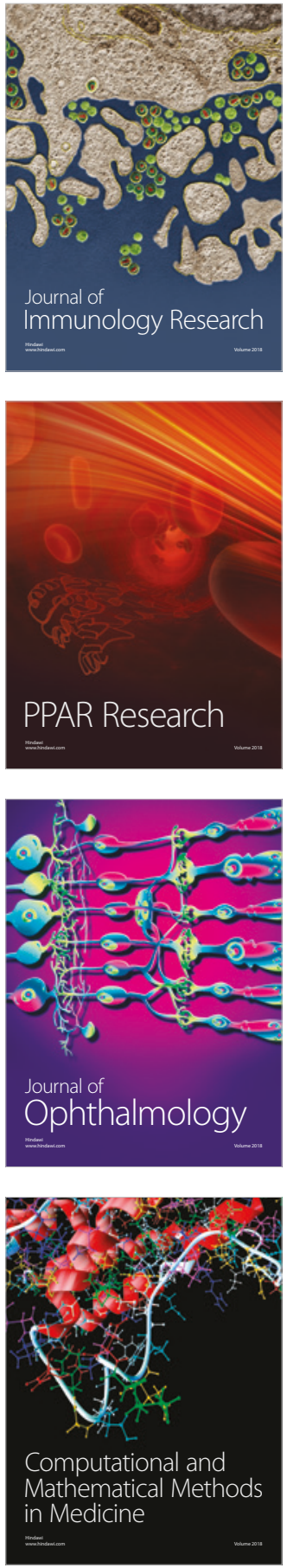

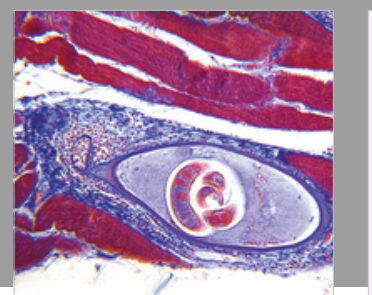

Gastroenterology Research and Practice

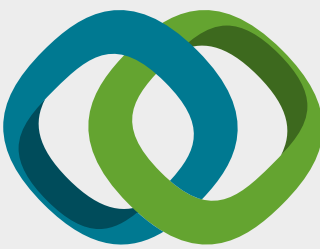

\section{Hindawi}

Submit your manuscripts at

www.hindawi.com
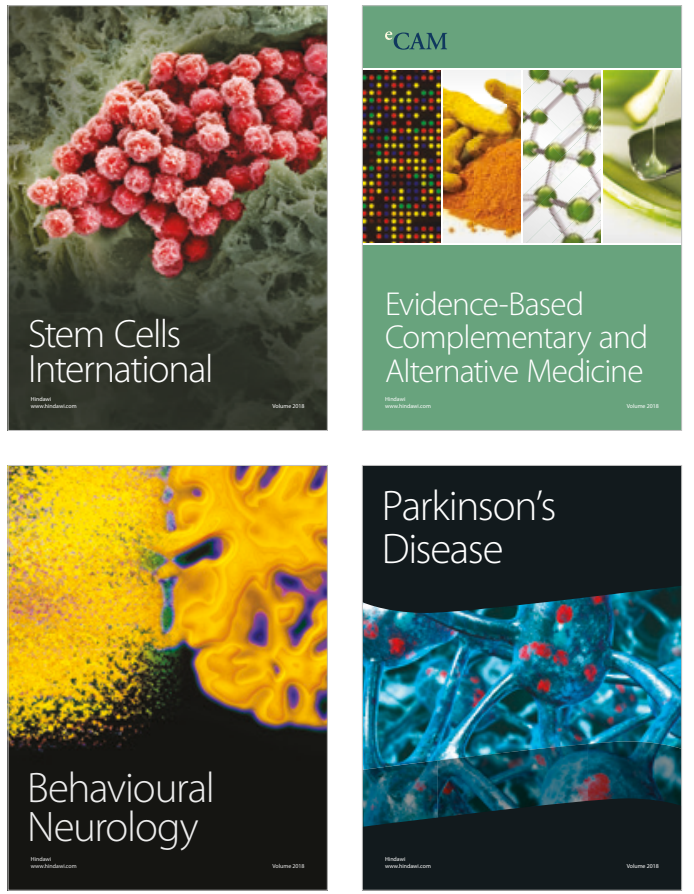

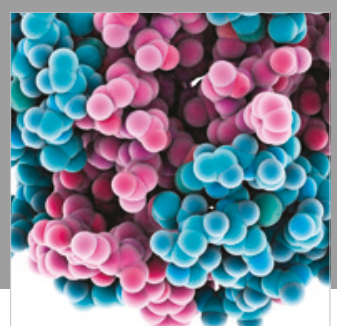

ournal of

Diabetes Research

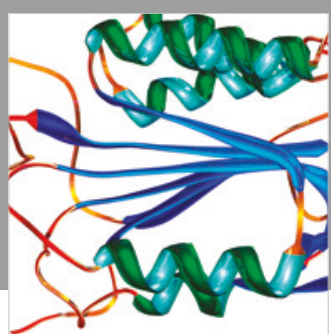

Disease Markers
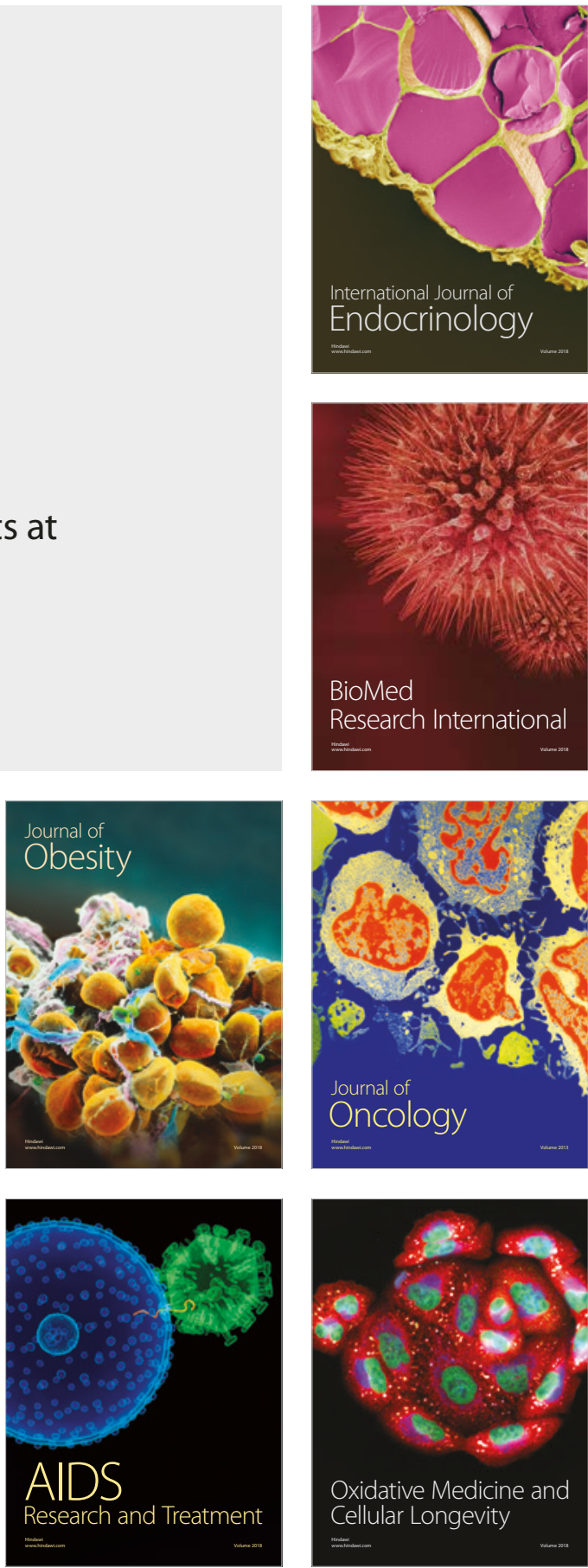